\title{
Multigene methylation analysis of enriched circulating tumor cells associates with poor progression-free survival in metastatic breast cancer patients
}

\author{
Theresa Benezeder ${ }^{1}$, Verena Tiran ${ }^{1}$, Alexandra A.N. Treitler $^{1}$, Christoph Suppan $^{1}$, \\ Christopher Rossmann ${ }^{1}$, Herbert Stoeger ${ }^{1}$, Richard J. Cote ${ }^{2}$, Ram H. Datar ${ }^{2}$, Marija \\ Balic $^{1,3}$ and Nadia Dandachi ${ }^{1,4}$ \\ ${ }^{1}$ Medical University of Graz, Department of Internal Medicine, Division of Oncology, Graz, Austria \\ ${ }^{2}$ University of Miami Miller School of Medicine, Department of Pathology, Miami, Florida, U.S.A. \\ ${ }^{3}$ Medical University of Graz, Research Unit Circulating Tumor Cells and Cancer Stem Cells, Graz, Austria \\ ${ }^{4}$ Medical University of Graz, Research Unit Epigenetic and Genetic Cancer Biomarkers, Division of Oncology, Graz, Austria \\ Correspondence to: Nadia Dandachi, email: nadia.dandachi@medunigraz.at \\ Marija Balic, email: marija.balic@medunigraz.at
}

Keywords: circulating tumor cells, enrichment, metastatic breast cancer, methylation, prognosis

Received: April 12, $2017 \quad$ Accepted: August 27, $2017 \quad$ Published: September 30, 2017

Copyright: Benezeder et al. This is an open-access article distributed under the terms of the Creative Commons Attribution License 3.0 (CC BY 3.0), which permits unrestricted use, distribution, and reproduction in any medium, provided the original author and source are credited.

\section{ABSTRACT}

Blood-based biomarkers such as circulating tumor cells (CTCs) provide dynamic real-time assessment of molecular tumor characteristics beyond the primary tumor. The aim of this study was to evaluate the feasibility of a size-based microfilter to assess multigene methylation analysis of enriched CTCs in a prospective proof-of principle study. We examined the quantitative methylation status of nine genes (AKR1B1, BMP6, CST6, HOXB4, HIST1H3C, ITIH5, NEUROD1, RASSF1, SOX17) in enriched CTCs from metastatic breast cancer patients. Feasibility and clinical performance testing were assessed in a test set consisting of 37 patients and 25 healthy controls. With established cut-off values from the healthy control group, methylation of enriched CTCs was detected in at least one gene in 18/37 patients $(48.6 \%)$, while $97.8 \%$ of all control samples were unmethylated. Patients with CTCs unmethylated for CST6, ITIH5, or RASSF1 showed significantly longer PFS compared to patients with corresponding enriched methylated CTCs. This proof-of-principle study shows the feasibility of a size-based microfilter to enrich and analyze multigene methylation profile of CTCs from metastatic breast cancer patients. For the first time, we report that multigene methylation analysis of enriched CTCs provides prognostic information in metastatic breast cancer patients.

\section{INTRODUCTION}

Breast cancer is the most frequently diagnosed cancer and the leading cause of cancer death among females worldwide, accounting for $25 \%$ of total cancer cases and $15 \%$ of all cancer deaths among females [1]. While chances of being cured of breast cancer have increased over the last decade, metastatic breast cancer remains essentially incurable and accounts for the majority of disease-related mortality [2]. Clearly, new prognostic and predictive biomarkers are needed in the metastatic setting. In this context, minimal-invasive blood-based biomarkers are a valuable source, providing important clinical and biological information of the tumor. In particular, blood-based biomarkers allow a real-time assessment of the molecular tumor genotype, thereby offering the possibility to monitor temporal and clonal evolution of tumor cells beyond the primary tumor. 
Tumor cells that have left the primary tumor or metastases can be found in the circulation of patients and these circulating tumor cells (CTCs) can provide unique biological and clinical information [3]. Several studies have clearly documented the prognostic value of CTCs in a number of solid tumor malignancies [4-9], and these blood-based biomarkers have been acknowledged as a liquid biopsy allowing the monitoring of disease progression and efficacy of cancer treatment [3]. Most of these studies have used the affinity-based CellSearch system (Janssen Diagnostics, NJ, USA), which is also the only technology approved by the US Food and Drug Administration for CTC enumeration [9-11]. However, an important and well-known limitation of the CellSearch platform is that enrichment is based on tumor cells expressing epithelial cell adhesion molecules (EpCAM). Subpopulations of cells that have low or absent EpCAM expression may be missed by this system. Importantly, acquisition of a mesenchymal phenotype has been shown to be a frequent characteristic of epithelial cancer cells during disease progression and metastasis [12] and this process is also accompanied by down-regulation of epithelial markers such as EpCAM [13-15].

Due to the low number of CTCs in blood, even in metastatic patients, detection and molecular characterization of CTCs remain challenging. To address these difficulties, a variety of techniques has been used for enrichment and detection of CTCs using cell surface markers or morphological differences between CTCs and normal blood cells $[16,17]$. In this project, we used an enrichment technique that captures CTCs based on their size and is independent of EpCAM expression on CTCs $[18,19]$.

With the increasing numbers of CTC studies, it has also become clear that beyond enumeration of CTCs, molecular characterization is necessary to better understand the role of CTCs in tumor progression and metastasis $[3,20,21]$. Importantly, a recent study indicates that CTCs are highly heterogeneous even within the same patient [22]. Taken together, studying CTCs has considerable potential to improve our current understanding of tumor heterogeneity and metastatic disease.

Altered DNA methylation is a well-established and frequent molecular hallmark of cancer cells. Particularly, hypermethylation of specific genes leads to gene silencing and contributes to the malignant phenotype [23]. Several genes have already been shown to be hypermethylated in breast cancer and some methylation profiles have also been associated with distinct breast cancer subtypes [24]. DNA methylation also occurs in genes involved in epithelial-mesenchymal-transition (EMT) [24], a process associated with tumor cell dissemination and metastasis. In most tumors, DNA methylation alterations are more frequent than genetic mutations, offering a wide range of potential targets [25].
So far, only few studies exist on DNA methylation of CTCs, but they already provide evidence for a potential biological and clinical role [26-29]. These studies mainly used antibody-based enrichment of CTCs and no study so far has evaluated size-based enrichment technologies in association with CTC methylation. Considering the accumulated evidence for the role of methylation in cancer progression and metastasis, further studies on the epigenetics of CTCs are definitively needed.

In this project, we used a size-based microfilter to enrich for CTCs with subsequent multigene methylation analysis of captured CTCs. We examined promoter methylation of nine candidate genes that are commonly methylated in breast cancer (AKR1B1, BMP6, CST6, HOXB4, HIST1H3C, ITIH5, NEUROD1, RASSF1, SOX17) $[26,30-32]$. To accurately quantify DNA methylation, we used pyrosequencing, which offers a sensitive and highly reproducible method [33].

\section{RESULTS}

\section{CTC enumeration}

Using our size-based microfilter, 37 samples were tested for the presence of CTCs identified as nucleated $\mathrm{CK}+/ \mathrm{DAPI}+/ \mathrm{CD} 45-$ cells. 19 out of 37 patients $(51.4 \%)$ had $>=1$ CTC per $7.5 \mathrm{ml}$ blood, with a median of 1 cell (range $0-56$ cells). 18 patients $(48.6 \%)$ had no CTCs, 10 patients $(27.1 \%)$ had $1-4$ CTCs and another 9 patients (24.3\%) had $>=5 \mathrm{CTCs} / 7.5 \mathrm{ml}$ blood.

\section{Analytical performance of pyrosequencing assays}

\section{Linearity, reproducibility, and limit of detection (LOD)}

We assessed the analytical performance of our pyrosequencing assays using a series of control samples. First, in order to test the linearity of the pyrosequencing assays, we generated dilution series consisting of unmethylated and fully methylated control samples at various percentages of methylation $(0 \%, 5 \%, 10 \%$, $25 \%, 50 \%, 75 \%$ and $100 \%$ ). We found a strong linear correlation between theoretical and observed methylation values for each gene as well as for the calculated CMI for all nine genes with $\mathrm{R}^{2}$ values ranging from 0.961 to 0.997, demonstrating that there is no amplification bias and methylated and unmethylated molecules are equally amplified. The generated dilution series were also used to calculate the limit of detection (LOD) for all nine assays according to Armbruster et al [34]. Calculated LOD values ranged from $1.1 \%$ to $4.1 \%$, limits generally reported for pyrosequencing assays [35]. As part of the quality control and in order to assess the robustness and reproducibility of pyrosequencing, we analyzed results from the same set of bisulfite-modified controls (MCF7, H1299 and MNCs) tested on different days in different pyrosequencing 
runs. The median of the gene-specific coefficient of variation $(\mathrm{CV})$ value for the methylated control samples (MCF7 and H1299) was $2.1 \%$ (range $0.5 \%$ to $4.2 \%$ ), and for the unmethylated control sample (MNCs) 10.1 (range $8.7 \%$ and $26.7 \%$ ). These data demonstrate a high analytical performance of all pyrosequencing assays with reproducible run-to-run variations of control samples allowing for accurate and reproducible quantification of DNA methylation.

\section{Assay validation using spiked-in breast cancer cells}

Next, blood samples from healthy controls were spiked with varying amounts of MCF7 (50-1000) cells and blood was then subjected to CTC enrichment with the size-based microfilter. Considering the analytical LOD of pyrosequencing (in general between 1 and 5\%), the lowest number of spiked cells was 50 cells in $7.5 \mathrm{ml}$ blood (corresponding to about 6 cells per ml blood). After CTC capture, DNA was extracted, bisulfite-converted and tested by pyrosequencing for the 9-gene panel, excluding HIST1H3C, which is not methylated in MCF7 cells. Calculated CMI values were higher in all samples spiked with MCF7 cells compared to the unspiked sample (Figure 1), demonstrating the technical feasibility of the size-based microfilter for multigene methylation analysis.

\section{Clinical performance of multigene CTC methylation analysis}

Finally, to verify the performance of our 9-gene panel for detecting methylation status of CTCs, we evaluated a clinical test set consisting of metastatic breast cancer patients $(n=37)$ and healthy controls $(n=25)$. Supplementary Table 2 summarizes all methylation results as well as CTC counts for each individual patient.

First, we assessed DNA methylation of all nine candidate genes in MNCs isolated from 25 healthy controls, representing the major source of DNA remaining on the microfilter. Quantitative methylation data from this control group was used to establish a cut-off for positive methylation according to Lehmann et al [36]. Table 1 shows the mean methylation values for all genes in the control group and the corresponding calculated cut-off values. These cut-off values were all higher than the calculated analytical LODs. Based on these cut-off values, $A K R 1 B 1, N E U R O D 1$ and $S O X 17$ were each methylated in $1 / 25(4.0 \%)$ control samples, HIST1H3C methylation was observed in $2 / 25(8.0 \%)$ control samples. All other genes (BMP6, CST6, HOXB4, ITIH5, and RASSF1) were unmethylated in the control group. Overall, $97.8 \%$ of all control samples were unmethylated.

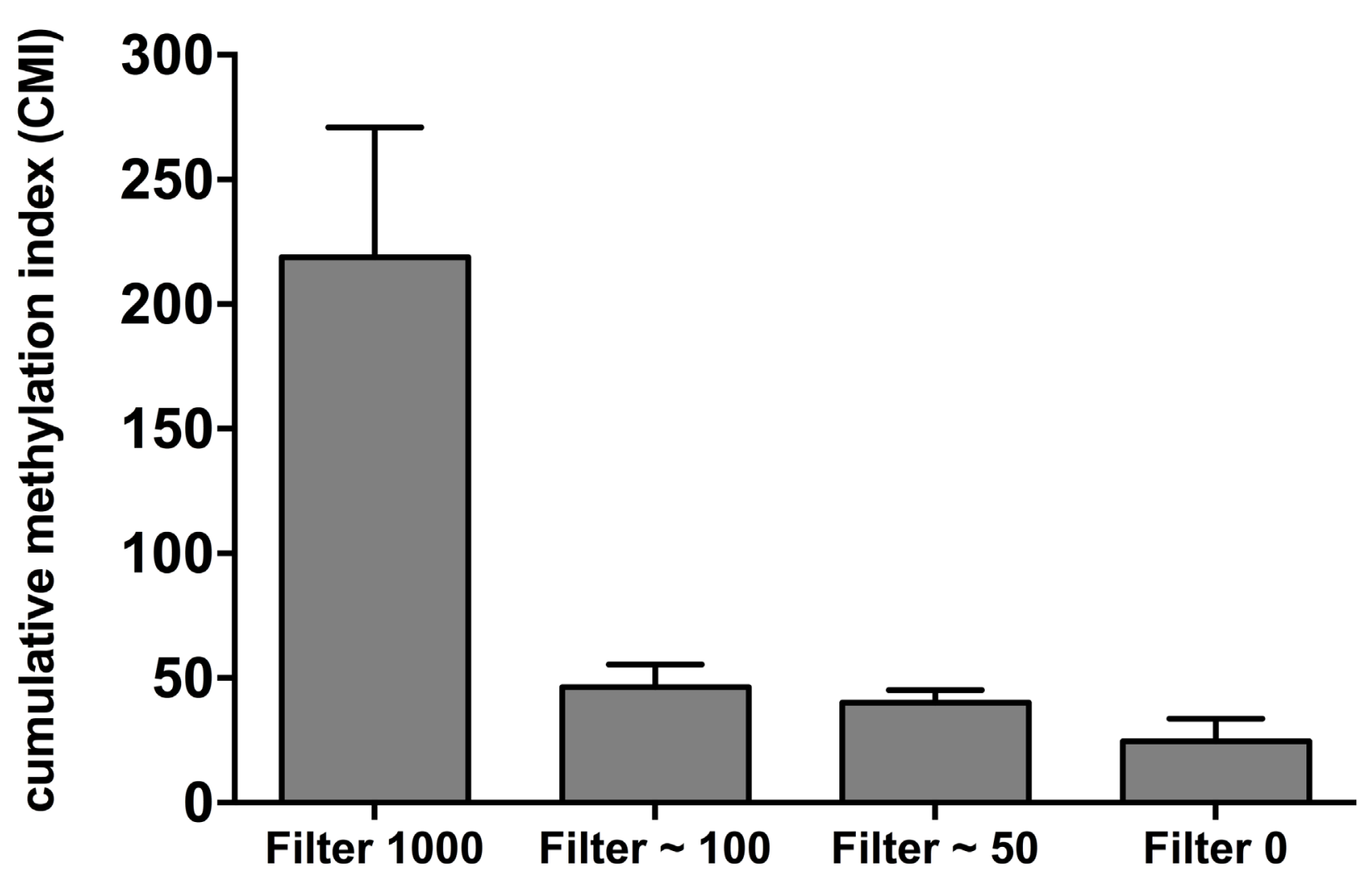

Figure 1: A cumulative methylation index (CMI) was calculated as the sum of the methylation percentage for the genes analyzed by spiking increasing numbers of MCF7 cells in $7.5 \mathrm{ml}$ healthy blood. CMI values were obtained from four independent spiking experiments and data are represented as mean $\pm \mathrm{SD}$. 
Table 1: DNA methylation levels in peripheral blood cells from healthy controls $(n=25)$ and corresponding cut-off values

\begin{tabular}{lccc}
\hline Genes & Mean methylation level & SD & Cut-off $^{*}$ \\
\hline AKR1B1 & 2.5 & 0.8 & 4.2 \\
BMP6 & 3.9 & 1.1 & 6.1 \\
CST6 & 5.1 & 0.9 & 6.9 \\
HIST1H3C & 4.7 & 1.8 & 8.3 \\
HOXB4 & 2.4 & 0.7 & 3.8 \\
ITIH5 & 4.7 & 1.1 & 6.8 \\
NEUROD1 & 5.3 & 0.9 & 7.1 \\
RASSF1 & 3.1 & 1.2 & 5.6 \\
SOX17 & 9.7 & 2.6 & 15.0 \\
\hline
\end{tabular}

${ }^{*}$ Cut-off defined as mean $+2 \mathrm{x}$ SD [35].

Next, we assessed the methylation status of enriched CTCs from metastatic breast cancer patients. Quantitative methylation results for each gene are shown in Figure 2. Using the established cut-off values from the healthy control group, methylation of CTCs was detected in at least one gene in $18 / 37$ patients $(48.6 \%)$. Of these positive samples, at least two genes were methylated in $61.1 \%$ of patients $(11 / 18)$, while only one gene was methylated in seven patients (38.9\%). Among all genes tested, ITIH5 (9/37) and HOXB4 $(8 / 37)$ were most frequently methylated in enriched CTCs, followed by CST6 (7/37) and RASSF1 (6/37). AKR1B1 was methylated in 3/37 patients, $S O X 17$ in 2/37 patients, and HIST1H3C and NEUROD1 were each only methylated in one of the 37 patients tested. PCR amplification failed for the $B M P 6$ gene in four patients despite repeating amplification twice, and was methylated in three of the 33 remaining patients. Overall, CST6, HOXB4, ITIH5 and RASSF 1 were more frequently methylated in CTCs from breast cancer patients compared to MNCs from healthy controls (Figure 3).

There was no association between positive CTC count and frequency of methylated genes in the CTC enriched cell fraction ( $p=0.330$, Fisher's exact test). In detail, out of 19 patients with $>=1$ CTC, 8 patients were unmethylated in all genes tested and 11 patients were methylated in at least one gene. On the other hand, out of 18 patients with no detectable CTCs, 11 patients showed no methylation and seven patients had at least one gene methylated. These results suggest that heterogeneous subpopulations of CTCs exist and that different subpopulations of CTCs may be identified using methylation profiling and $\mathrm{CTC}$ enumeration.

\section{Association between CTC methylation and clinicopathologic features}

Next, the methylation status of candidate genes was associated with known clinicopathologic characteristics, including age at blood sampling, tumor grade, tumor size, node status, ER, PR status, tumor subtype, location of metastases and number of metastatic sites. We found no significant associations between CTC methylation and hormone receptor status, HER2 status, node status, tumor subtype, number of metastatic sites or age (data not shown). Patients without liver metastasis had a significantly higher proportion of unmethylated $H O X B 4$ compared to patients with liver metastasis (Fisher's Exact Test, $p=0.021$ ). Additionally, patients with bone metastases were predominantly unmethylated with ITIH5 compared to patients without bone metastases (Fisher's Exact Test, $\mathrm{p}=0.042$ ). All other genes were not significantly associated with location of metastasis. Taken together, these results indicate that frequency of CTC methylation was not associated with classical clinical features of metastatic breast cancer patients in our study cohort.

\section{Clinical utility of CTC methylation analysis}

Finally, we evaluated the prognostic value of the most frequently methylated genes including CST6, HOXB4, ITIH5 and RASSF1, in enriched CTCs from 32 metastatic breast cancer patients. During the followup period 6 of 32 patients died and 18 of 32 patients progressed. The median follow-up time for the patients still alive at the end of the study was 338 days \pm 6.3 . In our study group, negative ER status and high tumor grade were associated with poor PFS in univariate analysis (data not shown). None of the classical prognostic parameters were associated with OS. This lack of significance for OS might be explained by the short follow-up time with a low number of death events (6/32 patients) in this study. Therefore, in subsequent univariate analysis we only focused on PFS. 
Methylation of CST6, ITIH5 and RASSF1 in enriched CTCs were significantly associated with shorter PFS in metastatic breast cancer patients. As shown in Figure 4, patients with methylated enriched CTCs, showed disease progression with a median PFS time of 168 days (CSTO), 260 days (ITIH5), and 77 days (RASSF1). In comparison, patients without CTC methylation demonstrated disease progression with a median PFS time of 331 days (CST6 and ITIH5), 312 days (RASSF1). Compared to patients without methylation, patients with enriched CTC methylation had a significantly higher risk of disease progression (Table 2). In contrast, methylation of $\mathrm{HOXB} 4$ was not significantly associated with PFS. Interestingly, CTC count was also not associated with PFS in our study group. CTC methylation of these genes also predicted shorter progression-free survival in the subgroup of patients with $\mathrm{HR}+/ \mathrm{HER} 2+$ phenotype $(\mathrm{n}=20)$ (data not shown).

Taken together, our data indicate that multigene methylation analysis of CTCs associates with poor PFS and thus may be useful in identifying patients at high risk for disease progression.

\section{DISCUSSION}

In this proof-of principle study, we could successfully demonstrate the feasibility of analyzing multigene methylation profiles of size-based enriched CTCs from patients with metastatic breast cancer. We used quantitative pyrosequencing to identify the methylation status of nine marker genes that have previously shown to be hypermethylated in breast cancer patients. We also provide evidence for the potential clinical value of CTC multigene methylation analysis, demonstrating an association between methylated enriched CTCs and poor progression-free survival.

Methylation alterations are established hallmarks in tumors and are more frequent than genetic mutations, providing a wide range of possible targets [23, 25]. So far, only few studies exist on CTC methylation, but they
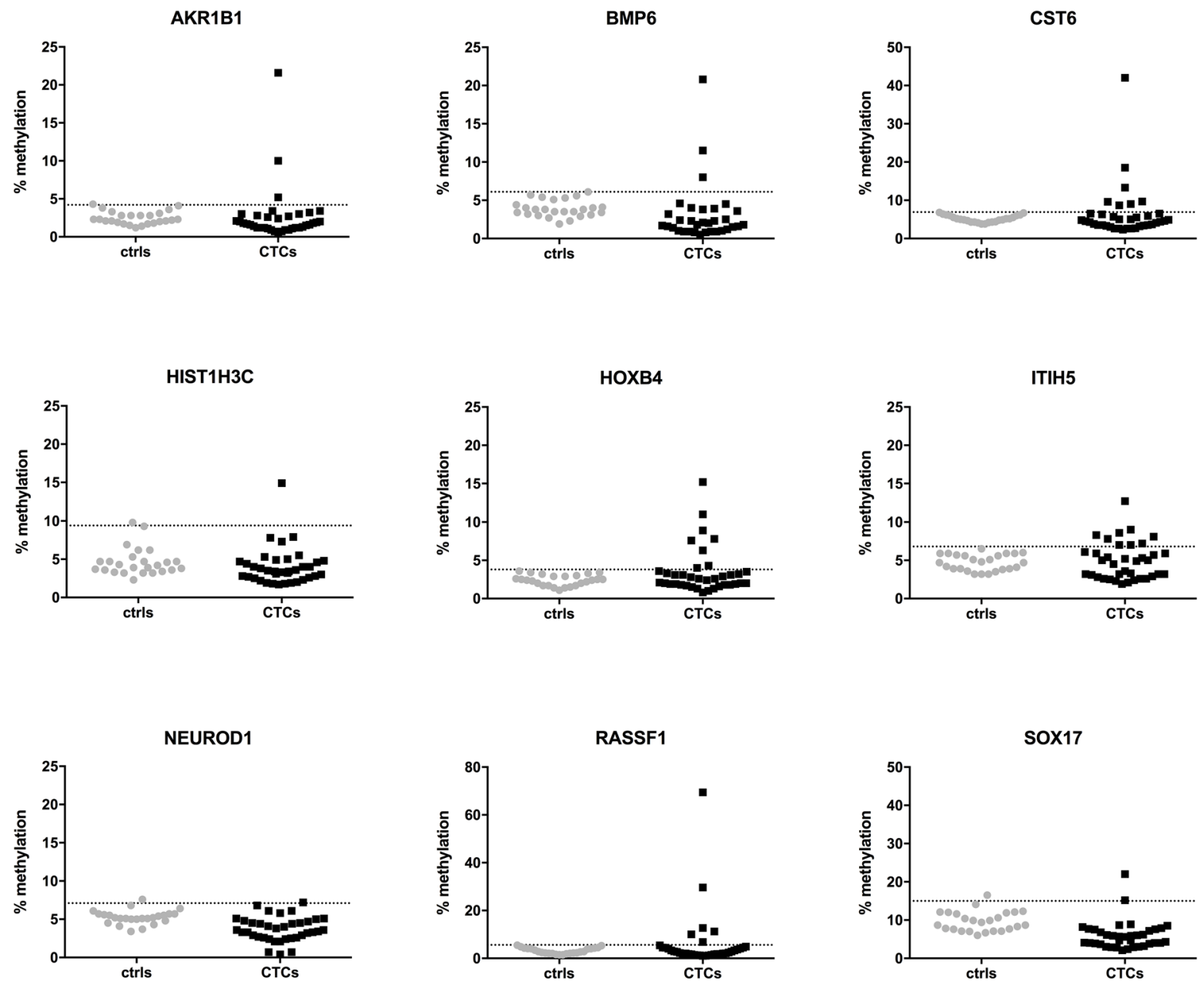

Figure 2: Quantitative methylation percentages of the 9 candidate genes analyzed by pyrosequencing in CTCs from metastatic breast cancer patients $(n=37$, black dots) and MNCs from healthy controls ( $\mathbf{n}=\mathbf{2 5}$, grey dots). The dotted horizontal line represents the cut-off for positive methylation. 
highlight first biological and clinical evidence of CTC methylation. Chimonidou and colleagues demonstrated in three studies that methylation status of three tumorassociated genes (CST6, BRMS1, and SOX17) can be detected in circulating tumor cells of breast cancer patients $[26,28,29]$. However, limitations of these studies were the use of semi-quantitative MSP and only a limited number of genes were analyzed.

To our knowledge, this is the first study to use a size-based enrichment platform and pyrosequencing to successfully assess multigene methylation profiling of CTC enriched cell fractions. The performance of the size- based microfilter to capture CTCs with a high efficiency has already been published elsewhere and was not within the scope of this study $[18,19]$. Here, we provide evidence that methylation levels can be detected in CTC enriched cell fractions after size-based filtration of blood samples from metastatic breast cancer patients. Overall, at least one gene was methylated in $18 / 37$ patients $(48.6 \%)$ and four out of 9 genes (CST6, HOXB4, ITIH5 and RASSF1) were more frequently methylated in the CTC enriched fraction compared to MNCs obtained from healthy individuals.

Following enrichment with a size-based microfilter, 19 patients with $>=1 \mathrm{CTC}$ were identified as $\mathrm{CK}+$ /

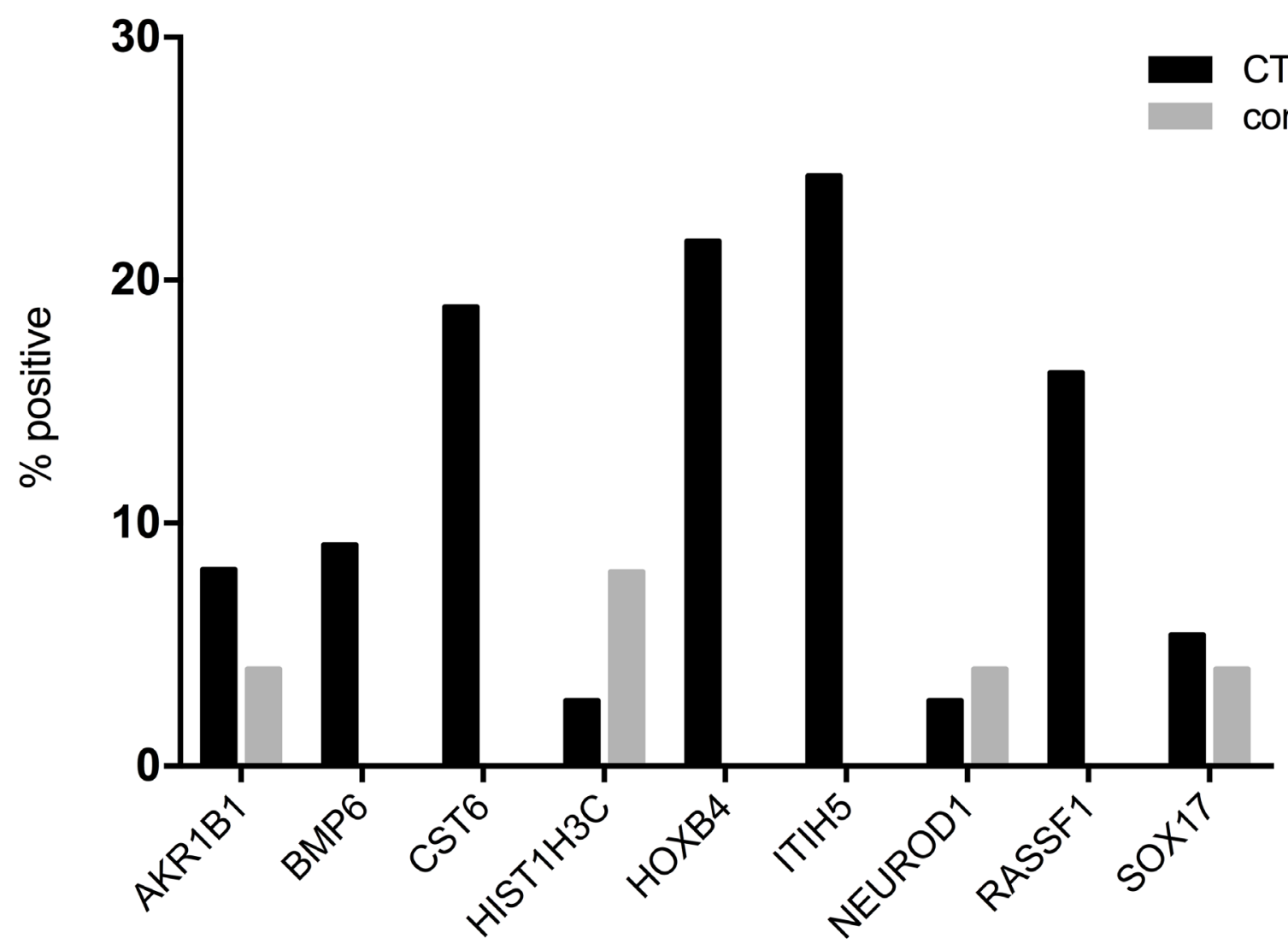

Figure 3: CST6, HOXB4, ITIH5 and RASSF1 are more frequently methylated in CTCs from metastatic breast cancer patients (black bars) compared to MNCs from healthy controls (grey bars). Samples were defined as positive when the mean methylation value for a sample was higher than the calculated cut-off value for the same gene.
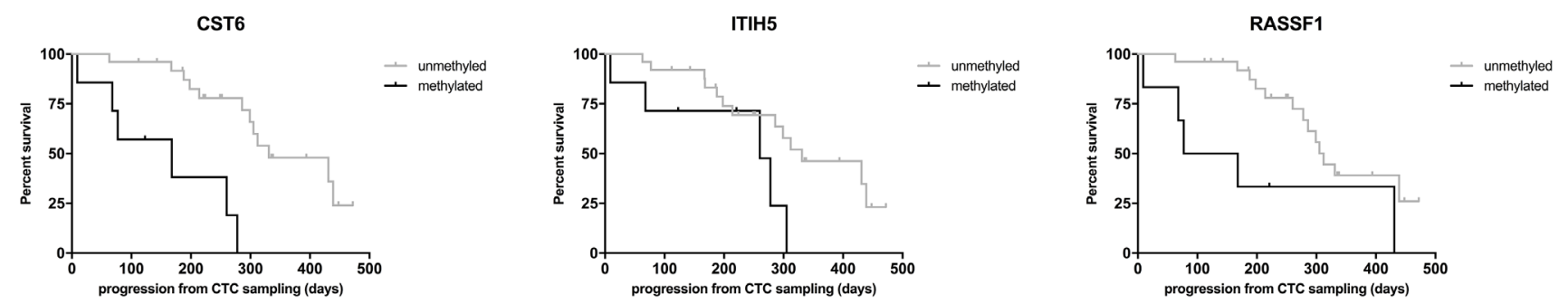

Figure 4: Impact of CTC methylation on progression-free survival (PFS) in metastatic breast cancer patients. PFS for patients with CST6, ITIH5, and RASSF1 methylation. black line, CTCs methylated, grey line, CTCs unmethylated. 
Table 2: Univariate Cox regression analysis of PFS

\begin{tabular}{lccc}
\hline Variable & $\mathbf{n}$ & HR $\mathbf{( 9 5 \%} \mathbf{C I})$ & P value \\
\hline CST6 methylation & 25 & 1.00 & \\
Negative & 7 & $7.99(2.33-26.84)$ & \\
Positive & 25 & 1.00 & \\
ITIH5 methylation & 7 & $2.93(0.97-8.88)$ & 0.058 \\
Negative & & & \\
Positive & 26 & 1.00 & \\
RASSF1 methylation & 6 & $3.17(1.10-9.17)$ & 0.033 \\
Negative &
\end{tabular}

HR hazard ratio, CI confidence interval.

DAPI+/CD45- cells. Out of these 19 CTC positive samples, only 10 samples showed methylation in at least one gene. Overall, we did not find any association between CTC counts and positive methylation. One reason for this finding could be that these CTCs identified as $\mathrm{CK}+/$ DAPI+/CD45- had other genes methylated than the ones used in this study or that both techniques identify different subsets of CTCs. Chimonidou et al also observed highly methylated genes in the CTC fraction with an EpCAMpositive/KRT19-negative phenotype [28], indicating the presence of different CTC subpopulations. Since our enrichment technique is independent of epithelial antigen expression, CTCs that have either lost the expression of antigens or have undergone EMT may not be identified by antibody-based enrichment methods and conventional enumeration based on CK expression, but may reveal methylation of tumor associated genes. In fact, a recent study of DNA methylation in single circulating tumor cells indicates tumor-specific epigenetic regulation of EMTassociated genes during blood-born dissemination [37].

CTCs are extremely rare, even in metastatic patients, which challenges and limits their detection and detailed characterization. This limitation was also evident in our study, demonstrating a relatively moderate sensitivity with $18 / 37$ (48.6\%) patients positive for at least one gene analyzed. While we used a multigene approach to account for the tumor heterogeneity, the number of samples available for methylation analysis remains limited. Furthermore, pyrosequencing has also a limited sensitivity as shown in this study, and more sensitive quantitative methods are necessary. Clearly, further technical improvements of CTC enrichment are also needed to provide a higher sensitivity allowing for a deeper insight into tumor heterogeneity at the CTC level. Analysis of additional genes could also help to improve the performance of this method.
While methylation of circulating free DNA (cfDNA) from plasma is also possible and perhaps more sensitive, our approach was designed to answer whether CTCs are methylated in breast cancer patients. Moreover, an important limitation of cfDNA is that its origin and more specifically tumor-specific alterations are not known and cannot be linked to specific tumor cell populations [38].

In this study, we used the pyrosequencing technique to assess methylation profiles of size-based enriched CTCs. Compared to the widely used semi-quantitative method MSP, pyrosequencing enables precise and sensitive quantification of DNA methylation [33]. Although highly sensitive, methods like MSP or QMSP are prone to generate false positive results and therefore overestimate the frequency of methylation events [39, 40]. In contrast, pyrosequencing not only facilitates the definition of cut-offs due to quantitative read-outs, but also offers the possibility to control for completeness of bisulfite treatment. This is a clear and essential advantage to many other methylation methods widely used.

Despite efficient CTC enrichment with the present microfilter [18, 19], a variable number of leukocytes still remains on the filter. Consequently, DNA isolated from the filter contains a mixture of both contaminating white blood cells and CTCs. However, except for the DEPArray $^{\mathrm{Tm}}$ system [41, 42], no other current CTC enrichment platform allows such pure capture of CTCs, and DEPArray ${ }^{\mathrm{Tm}}$ has its own limitations, including long hours to process and small blood volumes. To account for this limitation, we chose candidate genes that are not methylated in leukocytes. We confirmed the absence of methylation by analyzing MNCs of healthy individuals. All 9 genes were confirmed to be unmethylated in $97.8 \%$ of control samples consisting of MNCs and methylated to varying extents in five biologically different breast cancer 
Table 3: Clinical and pathological characteristics of metastatic breast cancer patients $(n=37)$

\begin{tabular}{l} 
Category \\
\hline Total \\
Age (years) at time of sampling
\end{tabular}

Median and range

\section{Menopausal status}

Premenopausal

Postmenopausal

Unknown

\section{Histologic type}

Invasive ductal/NST

Invasive lobular

Other

\section{Tumor grade (at primary diagnosis)}

Grade 1

Grade 2

Grade 3

Unknown

Primary tumor size

pT0/pT1

pT2

$\mathrm{pT} 3 / \mathrm{pT} 4$

Unknown

Lymph node status (at primary diagnosis)

NO

Unknown

Estrogen-receptor status (primary tumor)

Negative

Positive

Progesterone-receptor status (primary tumor)

Negative

Positive

\section{HER2 status (primary tumor)}

Negative

Positive 7

Unknown
18.9

5.4 


\begin{tabular}{|c|c|c|}
\hline Category & Number & $\%$ \\
\hline \multicolumn{3}{|l|}{ Subtype (primary tumor) } \\
\hline HR+HER2- & 25 & 67.6 \\
\hline HR-HER2- & 3 & 8.1 \\
\hline HER 2+ & 7 & 18.9 \\
\hline Unknown & 2 & 5.4 \\
\hline \multicolumn{3}{|c|}{ Metastatic location at time of sampling } \\
\hline Bone & 24 & 64.9 \\
\hline Lung & 15 & 40.5 \\
\hline Liver & 10 & 27.0 \\
\hline Other locations & 23 & 62.2 \\
\hline \multicolumn{3}{|l|}{$\begin{array}{l}\text { Number of metastatic sites at time of } \\
\text { sampling }\end{array}$} \\
\hline One & 16 & 43.2 \\
\hline Multiple & 21 & 56.8 \\
\hline \multicolumn{3}{|l|}{$\begin{array}{l}\text { Number of previous therapy lines for } \\
\text { metastatic disease }\end{array}$} \\
\hline 0 & 22 & 59.5 \\
\hline 1 & 7 & 18.9 \\
\hline$>=2$ & 8 & 21.6 \\
\hline \multicolumn{3}{|l|}{ Treatment initiated } \\
\hline Chemotherapy & 14 & 37.8 \\
\hline Chemotherapy and targeted therapy & 9 & 24.3 \\
\hline Hormone therapy & 13 & 35.1 \\
\hline No treatment & 1 & 2.7 \\
\hline
\end{tabular}

MBC metastatic breast cancer; NST not otherwise specified.

cell lines (data not shown). Furthermore, we applied a stringent cut-off value to define positive methylation using robust quantitative pyrosequencing technology. We also assessed methylation levels using blood from healthy volunteers $(\mathrm{n}=9)$ and after filtration of blood samples we found all candidate genes unmethylated (data not shown) in the CTC enriched cell fraction. Together, these data indicate that detected methylation levels result from the CTC enriched fraction on the filter, rather than from specific leukocyte subpopulations, which also remain on the filter.

Finally, we could show in our study that patients with enriched methylated CTCs for the genes CST6, ITIH5 and RASSF1 had a significantly shorter PFS time compared to patients with unmethylated CTC fractions. In contrast to gene methylation, CTC count did not reveal any prognostic value in our study cohort.
Limitations of this study include the small sample size, the heterogeneous breast cancer cohort (e.g. biological subtypes and number of previously received treatments) and the short follow-up time. These limitations could be one reason why CTC counts did not show any prognostic relevance. Clearly, our findings require validation in larger patient cohorts.

In conclusion, we show for the first time that tumor-specific methylation levels can be detected in CTC enriched cell fractions after size-based filtration of blood samples from metastatic breast cancer patients. Because this enrichment platform is independent of cell surface marker expression, capture of heterogeneous CTC subpopulations is possible. Our initial results also provide important evidence of the clinical utility of multigene methylation analysis of enriched CTCs in metastatic breast cancer patients. 


\section{MATERIALS AND METHODS}

\section{Patients and sample collections}

A total of 37 patients were enrolled in this prospective proof-of-principle study at the Division of Oncology, Department of Internal Medicine, at the Medical University of Graz. Blood samples were collected from patients with metastatic breast cancer at first diagnosis or at disease progression before starting a new line of systemic treatment. Patients with malignancies other than breast cancer were excluded. Blood samples were also collected from 25 healthy individuals. The study was approved by the local Institutional Review Boards (24539 ex 11/12), and all patients and donors gave their written informed consent. For cancer patients, $7.5 \mathrm{ml}$ of blood was drawn into CellSave tubes (Veridex LLC, Janssen Diagnostics, Rarities, NJ, USA), and for healthy donors, $9.0 \mathrm{ml}$ blood was collected in EDTA tubes (Greiner bioone, Gremsmünster, Austria). $0.225 \mathrm{ml}$ of a $10 \%$ neutralbuffered solution containing formaldehyde (4\% weight per volume, Sigma-Aldrich, Vienna, Austria) was added into EDTA tubes immediately after blood withdrawal.

Clinical and pathological data for metastatic breast cancer patients were retrieved from clinical records and are presented in Table 3. At the time of the blood draw, the age of the breast cancer patients ranged from 23 to 79 years with a mean of $58 \pm 13$. The control individuals were aged between 18 and $50(32 \pm 10)$. Five patients $(13.5 \%)$ were excluded for prognostic evaluation, because at the time of blood sampling, initial treatment had already started in four cases and one patient did not receive any treatment. Of the remaining 32 patients, disease progression was observed in 18 cases $(56.3 \%)$ and death in 6 cases (18.8\%). Median follow-up time was 338 days \pm 6.3 .

\section{Cell lines and control samples}

The MCF7 breast cancer cells and H1299 lung adenocarcinoma cells were obtained commercially from the American Type Culture Collection (ATCC, Manassas, USA) and cultured according to the supplier's recommendations and were used as control samples. Cell lines were authenticated by DNA short-tandem repeat analysis by the Cell Culture Facility of the Center for Medical Research at the Medical University of Graz (Austria). Fully methylated and unmethylated human control DNA (Zymo Research, Orange, CA, USA) were mixed to obtain following ratios of methylation: $0 \%, 5 \%$, $10 \%, 25 \%, 50 \%, 75 \%$ and $100 \%$.

Mononucleated blood cells (MNCs) were prepared from blood of healthy controls using Ficoll plaque density gradient centrifugation according to the manufacturer's instructions (Lymphoprep, Axis-Shield PoC, Oslo, Norway). MNCs at the interface were harvested and washed in phosphate-buffered saline (PBS). Cells were resuspended in $200 \mu \mathrm{PBS}$ and stored at $-20^{\circ} \mathrm{C}$ until DNA extraction.

\section{Spiking experiments}

MCF7 cells were counted with the Cellometer Auto 1000 (Nexcelom Bioscience, Lawrence, MA, USA) and the solution was then diluted in PBS to a final concentration of $1 \times 10^{5}$ tumor cells $/ \mathrm{ml}$. 50-1000 MCF7 cells were spiked into $7.5 \mathrm{ml}$ blood from healthy controls. Spiking of low numbers of MCF7 cells $(<100)$ was performed according to a protocol as previously published [43]. Briefly, $1 \mu 1$ of cell suspension $\left(1 \times 10^{5}\right.$ cells $/ \mathrm{ml}$ ) was transferred to an ultralow attachment 96well plate (Corning Inc, Corning, NY, USA) and cells were counted under the microscope. Counted cells were then immediately pipetted into $7.5 \mathrm{ml}$ of whole blood. The remaining cells in the 96-well plate were also counted and subtracted from the original count in order to more precisely estimate the total number of cells spiked into blood. In detail, the exact numbers for the 100 cell sample were 98, 111 and 88 cells and for the 50 cell samples 36, 62 and 56 cells. Samples were processed for CTC enrichment as described below.

\section{CTC enumeration}

Circulating tumor cells were captured using a sizebased microfilter device as previously described $[18,19]$. A total of $7.5 \mathrm{ml}$ blood was diluted 1:1 with $1 \mathrm{x}$ PBS and fixed with a final concentration of $1 \%$ formalin (SigmaAldrich, Vienna, Austria) for 10 minutes. After fixation, blood was processed through the microfilter at a constant flow rate of $75 \mathrm{ml} /$ hour using a motorized syringe pump. Following filtration, CTC identification and enumeration was done by double immunofluorescence staining including a pan-cytokeratin (CK) antibody and CD45 antibody. Filters containing CTCs were placed onto a glass microscope slide and blocked with blocking buffer consisting of 5\% normal goat serum (Life Technologies, Vienna, Austria) and 3\% Triton X-100 (Sigma Aldrich) at room temperature for 30 minutes. Next, samples were incubated with primary antibodies, mouse antiCD45 (Ready-to-use; DAKO, Glostrup, Denmark), and polyclonal rabbit anti-cytokeratin (1:300; DAKO) at room temperature for 1 hour. Samples were then incubated with secondary antibodies, goat anti-mouse Alexa 594 and goat anti-rabbit Alexa 488 (both 1:100, Life Technologies) at room temperature for 1 hour. Finally, samples were counterstained with 4, 6-diamidino-2-phenylindole (DAPI, Invitrogen) and mounted on coverslips with ProLong Gold Antifade mounting media (Life Technologies). The entire area of the microfilter was viewed under a confocal laserscanning microscope (Zeiss, Oberkochen, Germany), and CTCs were identified as nucleated $\mathrm{CK}+/ \mathrm{DAPI}+/ \mathrm{CD} 45$ cells. 


\section{DNA extraction}

After CTC enumeration, the stained microfilter was removed from the microscope slide by immersion into icecold PBS and the filter was then transferred to a QIAGEN Lyse\&Spin Basket placed within a $2 \mathrm{ml}$ microcentrifuge tube. DNA extraction from captured CTCs and isolated MNCs was performed using the QiampDNA micro Kit (Qiagen, Hilden, Germany) according to the instructions provided in the QIAGEN Lyse\&Spin Basket handbook. Briefly, filters were placed into Lyse\&Spin Baskets within a $2 \mathrm{ml}$ collection tube and $475 \mu \mathrm{l}$ ATL buffer was added. Next, $25 \mu$ proteinase $\mathrm{K}$ was added and the sample was incubated at $56^{\circ} \mathrm{C}$ for 1 hour with shaking at $900 \mathrm{rpm}$. After the incubation period, the tube was centrifuged at 13,000 rpm for $1 \mathrm{~min}$ and the flow-through fraction containing the nucleic acids was subjected to DNA isolation using the QIAamp DNA Micro Kit. One $\mu \mathrm{g}$ of carrier RNA was added to each sample and DNA was eluted in $2 \times 25 \mu 1$ elution buffer AE. Genomic DNA from cultured cells was extracted using the QIAmp DNA Mini Kit (Qiagen) according to the manufacturer's instructions. The DNA was dissolved in a final volume of $100 \mu l$ buffer and quantified using a NanoDrop ND 2000 spectrophotometer (Thermo Fisher Scientific, Bremen, Germany).

\section{Methylation analyses}

For methylation analysis, genomic DNA was subjected to bisulfite conversion using the InnuConvert Bisulfite Basic Kit (Analytik Jena, Jena, Germany) according to the manufacturer's instructions. For CTCs, $40 \mu \mathrm{l}$ (representing the entire extracted genomic DNA) and for cell lines and control samples 1000ng were used for bisulfite conversion. The purified bisulfite-converted DNA was eluted twice in $22 \mu \mathrm{l}$ volume and stored at $-20^{\circ} \mathrm{C}$ for further analysis. DNA methylation analysis of bisulfite-converted DNA samples was performed using pyrosequencing (Pyromark Q24) with PyroMark Q24 Advanced CpG Reagents (Qiagen). The PCR reaction was performed using the PyroMark PCR Kit (Qiagen) according to the protocol with $2 \mu$ l bisulfite-converted DNA and with an annealing temperature of $56^{\circ} \mathrm{C}$ and 45 cycles. Final concentration of primers was $0.2 \mu \mathrm{M}$ for all genes. PCR products were visualized on $2 \%$ agarose gels to verify amplification of a single band prior to pyrosequencing.

Human MCF-7 breast and H1299 lung cancer cell lines, and DNA from peripheral blood mononuclear cells of healthy individuals were used as methylated and unmethylated controls in each PCR run. Blank controls (without DNA template) were also amplified with each PCR reaction.

For following genes assays were designed using the Pyromark Assay Design Software Version 2 (Qiagen): AKR1B1, BMP6, CST6, HOXB4, HIST1H3C, ITIH5,
NEUROD1, RASSF1, SOX17. All of these genes have been previously shown to be hypermethylated in breast cancer patients $[30,44]$. Primer sequences are listed in Supplementary Table 1 . Between five and $15 \mathrm{CpG}$ sites located in the $\mathrm{CpG}$ islands of the promoter region were evaluated. All genes have been previously investigated for DNA methylation and primers were positioned to investigate the same or similar $\mathrm{CpG}$ sites $[26,30,31$, 44]. All designed assays include a non-CG cytosine in the region for pyrosequencing, as an internal control for complete bisulfite conversion.

Pyrosequencing results were evaluated using the PyroMark Q24 Advanced 3.0.0 software (Qiagen). Methylation data are presented as the percentage of the mean methylation in all $\mathrm{CpG}$ sites analyzed per gene. Each pyrogram was assessed for quality controls, including completeness of bisulfite-conversion and expected peak height and sequence. Data were only included in the analyses when all quality controls passed.

A sample was considered as hypermethylated when the mean methylation for the individual gene was higher than the cut-off for the same gene. The cut-off was defined as the mean methylation in the control group (MNCs of healthy controls) plus twice the standard deviation [36]. Additionally, for each individual sample, a cumulative methylation index (CMI) was calculated as the sum of the methylation percentage for the genes analyzed.

\section{Statistical analysis}

Statistical analyses and plotting of data were performed with GraphPad Prism 6.0 (GraphPad Software, San Diego, CA, USA) and SPSS 23 (SPSS, Inc., Chicago, IL, USA). P values $<0.05$ were considered significant. The Fisher's exact test was used to test whether differences between methylation positivity of CTCs and MNCs from controls were significant. Overall-survival (OS) was calculated from the date of blood sampling to the date of death. Progression-free survival (PFS) was the interval from the date of blood sampling to the date of clinical progression or death, whichever came first. Univariable survival analyses were performed using Kaplan-Meier plots and the log-rank test for comparisons. Hazard ratios (HR) were calculated for PFS by univariate Cox regression models with $95 \%$ confidence interval (CI). Methylation levels from unmethylated samples and lowlevel methylation samples $(5 \%)$ were used to calculate Limit of Detection (LOD) for each gene using the formula described by Armbruster et al [34].

\section{Abbreviations}

CTCs, circulating tumor cells; PFS, progression-free survival; EMT, epithelial-mesenchymal transition; EDTA, ethylene-diamine-tetra-acetic acid; MNCs, mononucleated blood cells; CK, pan-cytokeratin; DAPI, 4, 6-diamidino- 
2-phenylindole; CMI, cumulative methylation index; OS, overall survival; HR, hazard ratios; LOD, limit of detection; $\mathrm{CV}$, coefficient of variation; $\mathrm{ER}$, estrogen receptor, PR, progesterone receptor; HR, hormone receptor; MSP, methylation-specific PCR; QMSP, quantitative methylation-specific PCR; $A K R 1 B 1$, aldo-keto reductase family 1 member $\mathrm{B} ; B M P 6$, bone morphogenetic protein 6; CST6, cystatin E/M; EpCAM, epithelial cell adhesion molecule; HER2, erb-b2 receptor tyrosine kinase 2; HOXB4, homeobox B4; HIST1H3C, histone cluster $1 \mathrm{H} 3$ family member c; ITIH5, inter-alpha-trypsin inhibitor heavy chain family member 5 ; KRT19, keratin 19; NEUROD1, neuronal differentiation 1; RASSF1, Ras association domain family member 1 ; SOX17, SYR-box 17.

\section{Author contributions}

All the authors have accepted responsibility for the entire content of this submitted manuscript and approved submission. MB and ND designed the study and experiments and gave conceptual advice. TB and AT carried out methylation analysis and data interpretation. MB organized patient recruitment. CS and $\mathrm{CR}$ provided clinical data acquisition. HS provided clinical interpretation of data. VT performed CTC capture and identification. $\mathrm{RC}, \mathrm{RD}, \mathrm{TB}, \mathrm{MB}$ and $\mathrm{ND}$ gave conceptual advice and edited the manuscript. ND wrote the manuscript.

\section{ACKNOWLEDGMENTS}

We thank Klara Balic for English proofreading.

\section{CONFLICTS OF INTEREST}

The authors do not declare any conflicts of interest.

\section{FUNDING}

This research was supported by funds of the Oesterreichische Nationalbank, Anniversary Fund, project number 15358 (ND).

\section{REFERENCES}

1. Torre LA, Bray F, Siegel RL, Ferlay J, Lortet-Tieulent J, Jemal A. Global cancer statistics, 2012. CA Cancer J Clin. 2015;65:87-108.

2. Gennari A, Conte P, Rosso R, Orlandini C, Bruzzi P. Survival of metastatic breast carcinoma patients over a 20-year period: a retrospective analysis based on individual patient data from six consecutive studies. Cancer. 2005; 104:1742-50.
3. Bidard FC, Proudhon C, Pierga JY. Circulating tumor cells in breast cancer. Mol Oncol. 2016;10:418-30.

4. Bidard FC, Peeters DJ, Fehm T, Nolé F, Gisbert-Criado R, Mavroudis D, Grisanti S, Generali D, Garcia-Saenz JA, Stebbing J, Caldas C, Gazzaniga P, Manso L, et al. Clinical validity of circulating tumour cells in patients with metastatic breast cancer: a pooled analysis of individual patient data. Lancet Oncol. 2014;15:406-14.

5. Cristofanilli M, Hayes DF, Budd GT, Ellis MJ, Stopeck A, Reuben JM, Doyle GV, Matera J, Allard WJ, Miller MC, Fritsche HA, Hortobagyi GN, Terstappen LW. Circulating tumor cells: a novel prognostic factor for newly diagnosed metastatic breast cancer. J Clin Oncol. 2005;23:1420-30.

6. Hayes DF, Cristofanilli M, Budd GT, Ellis MJ, Stopeck A, Miller MC, Matera J, Allard WJ, Doyle GV, Terstappen LW. Circulating tumor cells at each follow-up time point during therapy of metastatic breast cancer patients predict progression-free and overall survival. Clin Cancer Res. 2006;12:4218-24.

7. Bidard FC, Vincent-Salomon A, Sigal-Zafrani B, Diéras V, Mathiot C, Mignot L, Thiery JP, Sastre-Garau X, Pierga JY. Prognosis of women with stage IV breast cancer depends on detection of circulating tumor cells rather than disseminated tumor cells. Ann Oncol. 2008;19:496-500.

8. Cohen SJ, Punt CJ, Iannotti N, Saidman BH, Sabbath KD, Gabrail NY, Picus J, Morse MA, Mitchell E, Miller MC, Doyle GV, Tissing H, Terstappen LW, Meropol NJ. Prognostic significance of circulating tumor cells in patients with metastatic colorectal cancer. Ann Oncol. 2009;20:1223-9.

9. Cristofanilli M, Budd GT, Ellis MJ, Stopeck A, Matera J, Miller MC, Reuben JM, Doyle GV, Allard WJ, Terstappen LW, Hayes DF. Circulating tumor cells, disease progression, and survival in metastatic breast cancer. N Engl J Med. 2004;351:781-91.

10. de Bono JS, Scher HI, Montgomery RB, Parker C, Miller MC, Tissing H, Doyle GV, Terstappen LW, Pienta KJ, Raghavan D. Circulating tumor cells predict survival benefit from treatment in metastatic castration-resistant prostate cancer. Clin Cancer Res. 2008;14:6302-9.

11. Cohen SJ, Punt CJ, Iannotti N, Saidman BH, Sabbath KD, Gabrail NY, Picus J, Morse M, Mitchell E, Miller MC, Doyle GV, Tissing H, Terstappen LW, Meropol NJ. Relationship of circulating tumor cells to tumor response, progression-free survival, and overall survival in patients with metastatic colorectal cancer. J Clin Oncol. 2008;26:3213-21.

12. Polyak K, Weinberg RA. Transitions between epithelial and mesenchymal states: acquisition of malignant and stem cell traits. Nat Rev Cancer. 2009;9:265-73.

13. Gorges TM, Tinhofer I, Drosch M, Röse L, Zollner TM, Krahn T, von Ahsen O. Circulating tumour cells escape from EpCAM-based detection due to epithelial-tomesenchymal transition. BMC Cancer. 2012;12:178. 
14. Joosse SA, Pantel K. Biologic challenges in the detection of circulating tumor cells. Cancer Res. 2013;73:8-11.

15. Rao CG, Chianese D, Doyle GV, Miller MC, Russell T, Sanders RA Jr, Terstappen LW. Expression of epithelial cell adhesion molecule in carcinoma cells present in blood and primary and metastatic tumors. Int J Oncol. 2005;27:49-57.

16. Yap TA, Lorente D, Omlin A, Olmos D, de Bono JS. Circulating tumor cells: a multifunctional biomarker. Clin Cancer Res. 2014;20:2553-68.

17. Alix-Panabières $\mathrm{C}$, Pantel K. Challenges in circulating tumour cell research. Nat Rev Cancer. 2014;14:623-31.

18. Lin HK, Zheng S, Williams AJ, Balic M, Groshen S, Scher HI, Fleisher M, Stadler W, Datar RH, Tai YC, Cote RJ. Portable filter-based microdevice for detection and characterization of circulating tumor cells. Clin Cancer Res. 2010;16:5011-8.

19. Zheng S, Lin H, Liu JQ, Balic M, Datar R, Cote RJ, Tai YC. Membrane microfilter device for selective capture, electrolysis and genomic analysis of human circulating tumor cells. J Chromatogr A. 2007;1162:154-61.

20. Lianidou ES, Markou A, Strati A. Molecular characterization of circulating tumor cells in breast cancer: challenges and promises for individualized cancer treatment. Cancer Metastasis Rev. 2012;31:663-71.

21. Ignatiadis M, Lee M, Jeffrey SS. Circulating tumor cells and circulating tumor DNA: challenges and opportunities on the path to clinical utility. Clin Cancer Res. 2015;21:4786-800.

22. Powell AA, Talasaz AH, Zhang H, Coram MA, Reddy A, Deng G, Telli ML, Advani RH, Carlson RW, Mollick JA, Sheth S, Kurian AW, Ford JM, et al. Single cell profiling of circulating tumor cells: transcriptional heterogeneity and diversity from breast cancer cell lines. PLoS One. 2012; 7:e33788.

23. Esteller M, Corn PG, Baylin SB, Herman JG. A gene hypermethylation profile of human cancer. Cancer Res. 2001;61:3225-9.

24. Stefansson OA, Moran S, Gomez A, Sayols S, ArribasJorba C, Sandoval J, Hilmarsdottir H, Olafsdottir E, Tryggvadottir L, Jonasson JG, Eyfjord J, Esteller M. A DNA methylation-based definition of biologically distinct breast cancer subtypes. Mol Oncol. 2015;9:555-68.

25. Baylin SB, Jones PA. A decade of exploring the cancer epigenome - biological and translational implications. Nat Rev Cancer. 2011;11:726-34.

26. Chimonidou M, Strati A, Malamos N, Georgoulias V, Lianidou ES. SOX17 promoter methylation in circulating tumor cells and matched cell-free DNA isolated from plasma of patients with breast cancer. Clin Chem. 2013;59:270-9.

27. Friedlander TW, Ngo VT, Dong H, Premasekharan G, Weinberg V, Doty S, Zhao Q, Gilbert EG, Ryan CJ, Chen WT, Paris PL. Detection and characterization of invasive circulating tumor cells derived from men with metastatic castration-resistant prostate cancer. Int J Cancer. 2014; 134:2284-93.

28. Chimonidou M, Strati A, Tzitzira A, Sotiropoulou G, Malamos N, Georgoulias V, Lianidou ES. DNA methylation of tumor suppressor and metastasis suppressor genes in circulating tumor cells. Clin Chem. 2011;57:1169-77.

29. Chimonidou M, Kallergi G, Georgoulias V, Welch DR, Lianidou ES. Breast cancer metastasis suppressor-1 promoter methylation in primary breast tumors and corresponding circulating tumor cells. Mol Cancer Res. 2013;11:1248-57.

30. Fackler MJ, Lopez-Bujanda Z, Umbricht C, Teo WW, Cho S, Zhang Z, Visvanathan K, Jeter S, Argani P, Wang C, Lyman JP, de Brot M, Ingle JN, et al. Novel methylated biomarkers and a robust assay to detect circulating tumor DNA in metastatic breast cancer. Cancer Res. 2014;74:2160-70.

31. Barekati Z, Radpour R, Lu Q, Bitzer J, Zheng H, Toniolo $\mathrm{P}$, Lenner P, Zhong XY. Methylation signature of lymph node metastases in breast cancer patients. BMC Cancer. 2012;12:244.

32. Chimonidou M, Tzitzira A, Strati A, Sotiropoulou G, Sfikas C, Malamos N, Georgoulias V, Lianidou E. CST6 promoter methylation in circulating cell-free DNA of breast cancer patients. Clin Biochem. 2013;46:235-40.

33. Marsh S, editor. Pyrosequencing applications. 1st ed. Vol. 373. New Jersey: Humana Press; 2007. 196 p.

34. Armbruster DA, Pry T. Limit of blank, limit of detection and limit of quantitation. Clin Biochem Rev. 2008;29:S49-52.

35. Lehmann U, Tost J, editors. Pyrosequencing. Vol. 1315. New York, NY: Springer New York; 2015. 1 p.

36. Lehmann U, Berg-Ribbe I, Wingen LU, Brakensiek K, Becker T, Klempnauer J, Schlegelberger B, Kreipe H, Flemming P. Distinct methylation patterns of benign and malignant liver tumors revealed by quantitative methylation profiling. Clin Cancer Res. 2005;11:3654-60.

37. Pixberg CF, Raba K, Müller F, Behrens B, Honisch E, Niederacher D, Neubauer H, Fehm T, Goering W, Schulz WA, Flohr P, Boysen G, Lambros M, et al. Analysis of DNA methylation in single circulating tumor cells. Oncogene. 2017;36:3223-31.

38. Pixberg CF, Schulz WA, Stoecklein NH, Neves RPL. Characterization of DNA methylation in circulating tumor cells. Genes (Basel). 2015;6:1053-75.

39. Mikeska T, Candiloro IL, Dobrovic A. The implications of heterogeneous DNA methylation for the accurate quantification of methylation. Epigenomics. 2010;2:561-73.

40. Brooks JD, Cairns P, Shore RE, Klein CB, Wirgin I, Afanasyeva Y, Zeleniuch-Jacquotte A. DNA methylation in pre-diagnostic serum samples of breast cancer cases: results of a nested case-control study. Cancer Epidemiol. 2010;34:717-23. 
41. Fabbri F, Carloni S, Zoli W, Ulivi P, Gallerani G, Fici P, Chiadini E, Passardi A, Frassineti GL, Ragazzini A, Amadori D. Detection and recovery of circulating colon cancer cells using a dielectrophoresis-based device: KRAS mutation status in pure CTCs. Cancer Lett. 2013;335:225-31.

42. Peeters DJ, De Laere B, Van den Eynden GG, Van Laere SJ, Rothé F, Ignatiadis M, Sieuwerts AM, Lambrechts D, Rutten A, van Dam PA, Pauwels P, Peeters M, Vermeulen PB, Dirix LY. Semiautomated isolation and molecular characterisation of single or highly purified tumour cells from CellSearch enriched blood samples using dielectrophoretic cell sorting. $\mathrm{Br} \mathrm{J}$ Cancer. 2013;108:1358-67.
43. Yoon HJ, Kim TH, Zhang Z, Azizi E, Pham TM, Paoletti C, Lin J, Ramnath N, Wicha MS, Hayes DF, Simeone DM, Nagrath S. Sensitive capture of circulating tumour cells by functionalized graphene oxide nanosheets. Nat Nanotechnol. 2013;8:735-41.

44. Kloten V, Becker B, Winner K, Schrauder MG, Fasching PA, Anzeneder T, Veeck J, Hartmann A, Knüchel R, Dahl E. Promoter hypermethylation of the tumor-suppressor genes ITIH5, DKK3, and RASSF1A as novel biomarkers for blood-based breast cancer screening. Breast Cancer Res. 2013;15:R4. 